\section{PO 8580 TREATMENT RESPONSE AMONG CAMEROONIAN ADOLESCENTS RECEIVING ANTIRETROVIRAL THERAPY IN URBAN AND RURAL SETTINGS: PRELIMINARY FINDINGS FROM THE READY STUDY}

'Joseph Fokam, 'Desire Takou²Maria Santoro, ${ }^{3}$ Armanda Nangmo, 'Samuel M Sosso, ${ }^{1}$ Georges Teto, ${ }^{2}$ Vittorio Colizzi, ${ }^{2}$ Carlo-Federico Perno, ${ }^{1}$ Alexis Ndjolo. ${ }^{1}$ Chantal BIYA International Reference Center for Research on HIVIAIDS prevention and management (CIRCB), Cameroon; ${ }^{2}$ University of Rome Tor Vergata, Rome, Italy; ${ }^{3}$ Faculty of Health Sciences, University de Bamenda, Cameroon

\subsection{6/bmjgh-2019-EDC.152}

Background Transitioning from paediatric to adult healthcare requires successful antiretroviral treatment (ART) for adolescents living with HIV (ADLHIV). Implementing such a policy implies monitoring ART response and selecting for therapeutic options for ADLHIV in resource-limited settings (RLS) like Cameroon.

Methods The Ready study (EDCTP-CDF-1027) is conducted amongst ART-experienced ADLHIV (10-19 years old) in the Centre region, Cameroon. WHO-clinical staging, CD4-counts and viraemia were determined; in case of virological failure [VF] (viraemia $\geq 1000$ copies/ml), HIV drug resistance (HIVDR) and subtyping were performed, and $\mathrm{p}<0.05$ considered significant.

Results Out of 279 ADLHIV (212 urban vs 67 rural), the gender distribution was similar $(54.5 \%$ female $)$; median age was higher in urban (15 [IQR: 13-17] years) compared to rural (13 [IQR: 11-17] years), as well as the median duration on ART (7 [IQR: 3-10] years compared to 4 [IQR: 2-7] years, respectively); and the majority was on first-line ART (79.4\% [162/204] urban vs 98.5\% [66/67] rural, p<0.0004). Following treatment response, clinical failure (WHO-stage 3/4) was similarly low in both urban $(5.7 \%$ [12/210]) and rural (4.5\% [3/67]), $p=0.938 ;$ CD4 increased similarly $(p=0.298)$ from ART-initiation (370 cells $/ \mathrm{mm}^{3}$ [urban] vs 332 cells/ $\mathrm{mm}^{3}$ [rural]) to 6 years after initiation $\left(938\right.$ cells $/ \mathrm{mm}^{3}$ [urban] vs 548 cells $/ \mathrm{mm}^{3}$ [rural]) and rate of immunodeficiency $(<500$ CD4 cells $\left./ \mathrm{mm}^{3}\right)$ was $41.0 \%(87 / 208)$ in urban vs $47.5 \%(29 /$ 61 ) in rural, $p=0.428$. VF was $43.2 \%$ (41/95) in urban vs $60.9 \%(14 / 23)$ in rural, $p=0.126$. Among nine (9) sequences available from those experiencing VF, overall HIVDR was found in $88.8 \%$, with $77.7 \%$ NNRTI, $55.6 \%$ NRTI and $22.2 \% \mathrm{PI} / \mathrm{r}$. All were HIV-1 group $\mathrm{M}$, with $55.6 \%$ CRF02_AG, 22.0\% F1 and 22.4\% others.

Conclusion ADLHIV appear clinically asymptomatic, with considerable immune recovery overtime. Despite differences in ART duration between urban and rural settings, VF was similarly high, associated with HIVDR mainly to NNRTI-based regimens. Thus, NNRTI-sparing regimens might be highly convenient when transitioning ADLHIV to adult ART-regimens in RLS like Cameroon.

\section{PO 8581 ZOONOTIC VIRAL ANTIGENS SURVEILLANCE IN HEALTHY POPULATIONS LIVING IN LAMBARÉNÉ, GABON}

${ }^{1}$ Emmanuel Bache* ${ }^{*}{ }^{1,2}$ Marguerite M Loembe, ${ }^{1,2}$ Selidji T Agnandji. ${ }^{1}$ Centre de recherches médicales de Lambaréné (CERMEL), Lambaréné, Gabon; ${ }^{2}$ Institut für Tropenmedizin, Universitätsklinikum Tübingen, Germany

10.1136/bmjgh-2019-EDC.153

Background Worldwide, viral zoonotic infections such as filoviruses, flaviviruses, nairoviruses and arenaviruses cause self- limiting to severe diseases. They are endemic in sub-Saharan Africa, causing sporadic outbreaks warranting the development of sustainable surveillance systems. In Gabon, Ebola outbreaks occurred from 1994 to 2002 causing 214 human cases and 150 deaths, while Dengue, Zika and Chikungunya virus outbreaks occurred between 2007 and 2010. Beyond these outbreaks, little is known about the epidemiology. Recently, in collaboration with the Japanese government, the Research and Health Ministries of Gabon supported the implementation of a biosecurity level-3 (BSL-3) laboratory at CERMEL in Lambaréné as a zoonotic disease surveillance unit. Start-off involved antigen detection and characterisation of circulating antibodies to targeted viral antigens in healthy populations. This study reports data from healthy participants (18-50 years) in a phase I rVSV-ZEBOV-GP Ebola vaccine trial.

Methods Hundred-six (106) baseline samples were screened for Ebola, Dengue (serotypes) 1-4 and Chikungunya viral RNA by RT-PCR on serum. IgG ELISA on plasma was used to identify antibodies against: Zaire-Ebola-(EBOV-GP and EBOV-VP40), Marburg-(MARV-GP and MARV-VP40), Crimean Congo Haemorrhagic Fever-(CCHFV-GP), Lasa-(LASV-GPC and LASV-NP), Yellow Fever-(YFV-NS1), West-Nile-(WNVNS1), Zika virus-(ZIKV-NS1), Chikungunya-(CHIKV-VLP) and Dengue-(DENV1-NS1,DENV2-NS1,DENV3-NS1,DENV4-NS1) virus antigens.

Results No viral RNA was isolated by RT-PCR in 106 samples. About 9\% (10/106), 3\% (3/106), 6\% (6/106), 24\% $(25 / 106), 51 \%(54 / 106), 38 \%(40 / 106)$ and $36 \%(38 / 106)$ participants were seropositive for antibodies specific to EBOV-GP, MARV-GP, CCHFV-GP, YFV-NS1, WNV-NS1, ZIKV-NS1 and CHIKV-VLP, respectively. Twelve percent $(12 \% ; 13 / 106)$ of participants possessed antibodies specific to Zika, Chikungunya and Dengue 1-4 antigens. Six percent $(6 \% ; 6 / 106)$ of participants were seropositive for EBOV-GP and CCHFV-GP.

Conclusion We found antibodies to viral zoonotic infections among our healthy volunteers. Further assays, including neutralisation assays are being performed to ascertain the specificity of the antibodies. These findings, once confirmed, will provide insights into disease surveillance, vaccine trial designs, evaluation of post-vaccine immune responses, variability in adverse events and overall disease transmission patterns.

\section{PO 8584 MULTIPLEXED MOLECULAR DETECTION OF MALARIA IN SIERRA LEONE}

1,2Rashid Ansumana*, 'Joseph M Lamin, 'Joseph Lahai, 'Umaru Bangura. 'Mercy Hospital Research Laboratory, Siera Leone; ${ }^{3}$ School of Community Health Sciences, Njala University, Sierra Leone

\subsection{6/bmjgh-2019-EDC.154}

Background Despite several control measures and policy changes in Africa, malaria remains one of the most prevalent diseases in West Africa. The gold standard for malaria diagnosis is microscopy. However, due to low technical capacities in resource-poor countries, rapid immunochromatographic tests are commonly used. In Sierra Leone, P. falciparum-specific ICT with histidine-rich proteins2(HRP-2) are used. HRP2 is specific to $P$. falciparum and the kit cannot be used to detect other species of malaria which are also present in the disease ecology in Sierra Leone.

Methods In this study, we assessed 182 febrile subjects for malaria between April 2017-July 2018 at the Mercy Hospital 
Research Laboratory in Sierra Leone. The blood samples collected were assessed using the Walter Reed Army Institute for Research(WRAIR) multiplex malaria PCR kit packaged by BioGX, Inc. (Alabama, USA) for detecting and speciation of malaria from human blood. Thin and thick slides were done for each sample and the images recorded by a digital scope. Results Results show that, out of 163 samples run by multiplex PCR for malaria, $81(49.7 \%)$ were positive for $P$. falciparum, while $82(50.3 \%)$ were positive for Plasmodium vivax. Conclusion The presence of $P$. vivax in the disease ecology without any significant difference $(\mathrm{p}>0.05)$ with $P$. falciparum poses problems for clinical outcomes of febrile illnesses. Panmalaria diagnostics in combination with $P$. falciparum could avert under-diagnosis of malaria.

\section{PO 8585 HIV, HBV AND HCV PREVALENCE, CO-INFECTIONS, RISK FACTORS AND AWARENESS AMONG STUDENTS IN A NIGERIAN UNIVERSITY}

${ }^{2}$ Margaret Japhet, ${ }^{1}$ Moses Adewumi, ${ }^{2}$ Adesina Olufisayo. ${ }^{1}$ Department of Virology, University College Hospital, University of Ibadan, Nigeria; '2Department of Microbiology, Faculty of Science, Obafemi Awolowo University, Nigeria

\subsection{6/bmjgh-2019-EDC.155}

Background HIV, Hepatitis B Virus (HBV) and Hepatitis C Virus (HCV) are life threatening viral infections. Co-infections are possible since they share routes of transmission through exchange of blood/body fluids. Youths are the most vulnerable to HIV infection due to unsafe practices. There is no free counselling and testing for $\mathrm{HBV} / \mathrm{HCV}$ in Nigeria, hence many may not be aware of their $\mathrm{HBV} / \mathrm{HCV}$ status. This study assessed prevalence, knowledge and risk factors of transmission among University students in order to provide preventive intervention.

Methods Previously counselled/consenting university students (total $=903, \mathrm{M}=502, \mathrm{~F}=428$; age range $16-40$ years; mean age 19 years) were enrolled. Relevant information was collected through questionnaire. About $5 \mathrm{ml}$ of blood was collected from each student and serum recovered was analysed for detectable HIV antigens/antibodies using specific ELISA kit. HIV antigen/antibody-positives were analysed for detectable hepatitis B surface antigen and anti-HCV. The HIV and HBVpositives were compared in terms of gender, age group, and risk factors by use of chi-square and Fischer exact tests, with two-tailed significance using SPSS version 20.0.1 for Windows. Result Of the 930 students examined, 630 (67.7\%) were sexually active and $104(16.5 \%)$ had multiple sex partners. Knowledge of HIV, HBV and HCV status was 55\%, 36.3\% and $4.2 \%$ respectively. Overall, $13(1.40 \%)$ students had detectable HIV antigens and/or antibodies, 5 (38.5\%) of whom were HBV-positive, none had HCV infection. All HBV-positive students were ignorant of their HBV status. HIV and HBV-positive students fall within age range 15-24 years with higher $\mathrm{HIV} / \mathrm{HBV}$ prevalence in females than males. Statistical significance exists between HIV, HBV prevalence and a) gender, b) number of sex partners, and c) sharing sharps with people of unknown HIV/HBV status $(\mathrm{p}=0.005 ; 0.002$ and 0.005 , respectively).

Conclusion Knowledge about HBV and HCV is generally low among the students. Awareness campaigns specifically tailored towards educating young adults on HIV, HBV and HCV prevention/control should be encouraged.

\section{PO 8590 COMPARATIVE ANALYSIS OF IGG RESPONSES TO RECOMBINANT Q $\beta$ PHAGE DISPLAYED MSP3 AND UBO5 IN DUAL HIV/MALARIA-CO-INFECTED ADULTS}

${ }^{1}$ Godwin Nchinda*, ${ }^{2}$ Abel Lissom, ${ }^{3}$ Herve Ouambo, ${ }^{4}$ Malachy I Okeke, ${ }^{5}$ Thibeau F Tchouangueu, ${ }^{6}$ Loveline N Tchouangueu, ${ }^{7}$ Arinze S Okoli, ${ }^{3}$ Eric Achidi, ${ }^{8}$ Wilfred Mbacham, ${ }^{9}$ Lazare Kaptue, ${ }^{10}$ Charles 0 Esimone, ${ }^{11}$ Rose F Leke, ${ }^{12}$ Alain B Waffo. ${ }^{1}$ Laboratory of Vaccinology/Biobanking CIRCB, Cameroon; '2Department of Animal Biology and Physiology, University of Yaoundé I, Cameroon; ${ }^{3}$ Department of Medical Laboratory Sciences, University of Buea, Cameroon; ${ }^{4}$ Molecular Inflammation Research Group, Department of Medical Biology, Faculty of Health Sciences, UiT The Arctic University of Norway, Norway; ${ }^{5}$ Department of Biochemistry, University of Dschang, Cameroon; ${ }^{6}$ Department of Biochemistry University of Yaounde One; 'GenØk - Centre for Biosafety, Tromsø, Norway; ${ }^{8}$ The Department of Biochemistry and Physiology, Faculty of Medicine, University of Yaoundé I, Cameroon; ${ }^{9}$ Université des Montagnes, Bangangté, Cameroon; ${ }^{10}$ Department of Pharmaceutical Microbiology and Biotechnology, Nnamdi Azikiwe University Awka, Nigeria;

${ }^{11}$ The Biotechnology Center, University of Yaoundé I, Cameroon; ${ }^{12}$ Department of Biological Sciences/College STEM 1627 Hall Street Montgomery, USA

\subsection{6/bmjgh-2019-EDC.156}

Background Immunoglobulin G (IgG)-specific responses against Plasmodium falciparum merozoite antigens such as the merozoite surface protein 3 (MSP3) and UBO5 are known to play critical roles in parasitaemia control and protection from symptomatic illness. However, when there is intense perennial malaria transmission coupled with concurrent infection with the human immunodeficiency virus type 1 (HIV), knowledge of IgG antibody response profiles is limited.

In this study we assessed the impact of dual HIV/malaria infections on IgG subclass responses to MSP3 (QBMSP3) and UBO5 (Q $\beta$ UB05) in individuals living in two areas of Cameroon differing in malaria transmission intensity.

Methods IgG and IgG subclass responses specific to either MSP3 or UBO5 were determined in plasma from study participant by ELISA. To improve reactivity with their respective antibodies the antigens were displayed upon the surface of the RNA coliphage Q $\beta$.

Results We observed differences in antigen-specific IgG and IgG subclass responses which were dependent upon the antigen type, malaria transmission intensity, HIV infection, malaria infection and dual HIV/malaria infections. Individuals living in areas with high malaria transmission, had irrespective of HIV or malaria status significantly higher $\operatorname{IgG}$ responses to both antigens $(p=0.0001$ for Q $\beta M S P 3, p=0.0001$ for $Q \beta U B 05)$ than their counterpart from areas with low transmission. When dual HIV/malaria infection is considered, significantly higher QßMSP3 specific IgG1 $(p=0.0001)$ and $\operatorname{IgG3}(p=0.04)$ responses in double-negative individuals was associated with protection against malaria in areas with low transmission. Superior QßUBO5 specific IgG1 responses $(p=0.0001)$ in double-negative individuals were associated with protection in areas with high transmission in contrast to significantly higher IgG3 responses to Q $\beta$ UBO5 $(p=0.0001)$ which were more relevant to protection in areas with low malaria transmission in the same population.

Conclusion Thus, understanding immune responses to QßUBO5 and QßMSP3 could facilitate the development of immunotherapeutic strategies suitable for areas differing in malaria transmission intensity. 